(c) 2010 IEEE. Personal use of this material is permitted. Permission from IEEE must be obtained for all other uses, in any current or future media, including reprinting/republishing this material for advertising or promotional purposes, creating new collective works, for resale or redistribution to servers or lists, or reuse of any copyrighted component of this work in other works. 


\title{
LCART: A Cross-layered Transport Protocol for Heterogeneous WSN
}

\author{
Atif Sharif, Vidyasagar M. Potdar and A.J.D Rathnayaka \\ DEBII, \\ Curtin University of Technology \\ Perth, WA \\ Email: \{a.sharif, v.potdar, a.rathnayaka\}@ curtin.edu.au \\ wsn.debii.curtin.edu.au
}

\begin{abstract}
Lightweight Congestion Aware Reliable Transport protocol (LCART) is based on cross-layering the prevalent or reciprocal functionalities of Transport, MAC and Wireless-Physical layers in order to achieve energy efficiency and meeting QoS objectives of heterogeneous $\mathrm{WSN}^{1}$ including multimedia. LCART intelligently overcomes network congestion by the simultaneous use of Packet Service Time, Packet Inter Arrival Time, Buffer Occupancy Level and Channel Loading threshold limits and ensures packet level reliability by the use of $\beta$ parameter entirely being dictated by the nature of traffic flow. LCART has been evaluated against TCP-Westwood+ (TCP-WW+), TCPWestwood (TCP-WW), TCPNewReno and TCPReno for 24 mote ad-hoc topology. The results reveal that LCART outperforms others by exhibiting highest good throughput of $0.3112 \mathrm{Mbps}$, average Endto-End (E-2-E) packet latency of $<80 \mathrm{msec}$ for multimedia and $<130$ msec for scalar information, $1.014 \%$ average percentage packet drop and overall exhibits energy efficient behavior.
\end{abstract}

\section{INTRODUCTION}

The use of Wireless Sensor Network (WSN) for heterogeneous traffic environment has attracted the intention of research community recently. This multi disciplinary technology used for gathering heterogeneous information, scalar or multimedia, has always been question marked for its power efficiency [1]. The transport layer protocol for heterogeneous WSN has gained fundamental importance for ensuring the congestion control and data reliability within WSN together with nature specific traffic flow for achieving the QoS objectives governed by the particular heterogeneous application [1]. Presently the transport layer protocols are designed either by targeting the protocol efficiency or to address the range of application scenarios where the protocol efficiency is comprised. Keeping this fact in mind, the researchers recently has come up with a new dimension of hybrid transport layer protocol designing also called as "Cross-layering" [2-3] which inherent the flavors of energy efficiency and addressing the range of application scenarios. Majority of the transport protocols for heterogeneous WSN like $\mathrm{RT}^{2}$ [4], RCRT[5], CTCP[6], FLUSH[7] etc provides packet level reliability in upstream direction and uses ACK, SACK and NACK for ensuring reliability. Also these protocols uses Packet Service Time (TPST), Packet Inter Arrival Time (TPIAT), Buffer Occupancy

\footnotetext{
${ }^{1}$ throughout the paper it represents the mixed traffic scenario including the multimedia information flow e.g. audio, video.
}

Level $\left(m_{i}\right)$ and Channel Loading threshold limits $\left(\lambda_{\text {Threshold }}\right)$ in discrete isolated fashion, not simultaneous, for explicit or implicit congestion notification within WSN. Except $\mathrm{RT}^{2}$, which cross-layered the Transport layer functionality with the Routing layer, rest all does not utilize this approach for gaining network efficiency while complying with the stringent QoS objectives specific to heterogeneous WSN in an energy efficient manner. We have observe the dependency of transport layer over underlying MAC and Wireless-physical layers [3] and based on this we are here envisaging a cross-layered approach for transport protocol named as 'LCART' which is based on the idea of Cross-layering, showing energy efficient behavior while addressing the range of application scenarios. Thus the motivation of LCART is to best utilize the crosslayer functionality to gain maximum energy efficiency. The rest of the paper is organized as following. After introduction the proposed transport layer protocol scheme is described in Section 2 followed by Section 3 where we have describe the simulation setup used for observing its behavior and the simulation results we have taken. The discussion followed by the conclusions will be presented in the last Section 4.

\section{PROPOSED TRANSPORT PROTOCOL: LCART}

This section will describe the proposed LCART for heterogeneous WSN which is based on the cross-layering approach as shown in the Figure 1. The detailed explanation of the LCART is outlined in the following subsections below:

\section{A. Protocol Overview}

LCART looks after the system throughput, mote's energy budget, E-2-E data packet latency and data packet drop by having two control loops running in concurrent:

E-2-E sink enabled feedback control loop monitors the E-2E data packet latency and the control loop triggers the source motes to readjust their transmission rates thereby minimizing the number of data packets actually suffering from an unwanted queue delay caused by congestion.

Whereas the Local Intermediate buffer mote enabled feedback loop monitors the TPIAT, TPST, channel conditions in its vicinity and local $m_{i}$. It then informs sink mote about these monitored statistics which is helpful in computing the new optimal source transmission rate plan. This efficiently 
exploits the network resources while minimizing the inter-hop packet delay and its drop caused by collisions, bad channel conditions and congestion. Other than this it is also responsible for the rapid data packet retrieval, in an Hop-by-Hop fashion, during the events of data packet loss caused by either condition discussed above.

The proposed scheme is comprised of Congestion Control, Packet Reliability and Data Prioritization modules for Heterogeneous WSN.

1) Congestion Control: The purpose of this module is to effectively control the congestion in order to minimize the packet drop due to congestion and to achieve optimum system throughput. This design module also computes the maximum effective load, by avoiding congestion, that a mote can put on a channel. For a given source mote having sending rate in Mbps $\lambda_{e}$, duty cycle of $\rho, e_{j}$ being the transmission error and probability of successful delivery to next hop $p$ then the $\lambda_{\text {Threshold }}$ is given by:

$\lambda_{\text {Threshold }} \leq \frac{\left[\rho-p \cdot\left(1+e_{j}\right) \cdot \lambda_{e}\right]}{\left[\left(1+e_{j}\right) \cdot p+1\right]}$

When the network alives for the first time, LCART computes the initial source transmission rate plan for various sources with the help of 3 control packets transmitted in upstream towards sink. After knowing TPST, TPIAT and $\lambda_{\text {Threshold }}$, the sink computes the initial source transmission rate $x_{0}$ (for all sources) in terms of packets per second and is given by:

$$
x_{0}=\min \left(T_{P S T}, T_{P I A T}, \lambda_{\text {Threshold }}\right)
$$

Now we will discuss the optimal solution for updating mote's transmission rate, sink enabled for easing congestion within network having ' $N$ ' motes distributed in space with ' $n$ ' neighboring motes to any particular mote. The solution is based on the Robust Kalman estimator (Predictor and Corrector Estimator) with the intention to have minimal processing over head and to gain significant network efficiency.

Predictor/Time update helps in estimating the source transmission rate and error covariance at current discrete interval $k$ based on the a priori statistics of TPST, TPIAT and $m_{i}$. So incorporating the system dynamics the Time Update equation for error covariance and source transmission rate would be:

$$
\begin{aligned}
& P_{k+1}^{-}=A_{k} P_{k} A_{k}^{T}+G_{k} Q_{k} G_{k}^{T} \\
& \hat{x}_{k+1}=\left[C_{k}-A_{k}\right] \hat{x}_{k}+B_{k} U_{k} \hat{x}_{k}
\end{aligned}
$$

where,

$$
\begin{aligned}
A_{k} & =\left[\begin{array}{c}
m_{i}\left(i, j_{1}\right) \\
m_{i}\left(i, j_{2}\right) \\
\cdot \\
m_{i}\left(i, j_{n}\right)
\end{array}\right], B_{k}=G_{k}=\left[\begin{array}{ccccc}
1 & 0 & \cdot & \cdot & 0 \\
0 & 1 & \cdot & \cdot & 0 \\
\cdot & \cdot & \cdot & \cdot & \cdot \\
\cdot & \cdot & \cdot & \cdot & \cdot \\
0 & 0 & \cdot & \cdot & 1
\end{array}\right] \\
C_{k} & =\left[\begin{array}{c}
1 \\
1 \\
\cdot \\
\dot{1}
\end{array}\right], U_{k}=\left[\begin{array}{c}
U_{k}\left(i, j_{1}\right) \\
U_{k}\left(i, j_{2}\right) \\
\cdot \\
U_{k}\left(i, j_{n}\right)
\end{array}\right]
\end{aligned}
$$

and $U_{k}(i, j)=\epsilon_{k}(i, j)+\gamma_{k}(i, j)$

where,

$\epsilon_{k}(i, j)=T_{P S T}^{\prime}-T_{P S T}$ and $\gamma_{k}(i, j)=T_{P I A T}^{\prime}(i, j)-T_{P I A T}(i, j)$

Similarly for sink, the incoming data or actual output of the system is given by:

$z_{k}=H_{k} x_{k}+v_{k}$

where,
Table 1: NETWORK PARAMETERS

\begin{tabular}{|c|c|}
\hline Parameter & Values \\
\hline \hline Frequency $(\mathrm{Hz})$ & $914 e^{+6}$ \\
\hline RX and CS & $3.6252 e^{-10} \&$ \\
Threshold (W) & $1.559 e^{-11}$ \\
\hline Ifqlen & 50 packets \\
\hline Mote Initial \& & $100,712 e^{-6}$ \\
Idle power (W) & \\
\hline Mote Rx \& Tx & $35.28 e^{-3}$, \\
power $(\mathrm{W})$ & $31.32 e^{-3}$ \\
\hline$v_{k} \& \rho$ & $0 \& 1$ \\
\hline$e_{j}$ & $1 \%$ \\
\hline$\beta$ & $0<\beta<1$ \\
\hline \multicolumn{2}{|c}{}
\end{tabular}

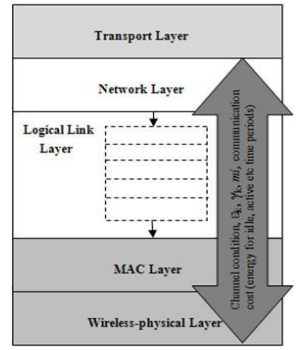

Figure 1. The Cross-layered Approach

$z_{k} \in R^{t}, G_{k}$ and $H_{k}$ are simple scaling matrix and,

$v_{k} \in R^{t}$ is the measurement noise (if any).

Corrector/Measurement update helps in (feedback to measurement update at $k+1$ interval) correcting the error covariance and source transmission rate by taking into account the effect of measurement $z_{k}$ and is given by:

$$
\begin{aligned}
& P_{k+1}=\left[\left(P_{k+1}^{-}\right)^{-1}+H_{k+1}^{T} R_{k+1}^{-1} H_{k+1}\right]^{-1} \\
& \text { and } \\
& \hat{x}_{k+1}=\hat{x}_{k+1}^{-}+P_{k+1} H_{k+1}^{T} R_{k+1}^{-1}\left(z_{k+1}-H_{k+1} \hat{x}_{k+1}^{-}\right)
\end{aligned}
$$

The sink computes and informs the new transmission rate plan to every source.

2) Packet Reliability: For multimedia traffic scenario, the packet based reliability is being dictated by the application specific QoS $\beta$ parameter. $\frac{20 * R T T}{\beta}$ defines the time in msec to retain a packet based on the nature of flow. The purpose of this module is to ensure the packet level reliability for both data packets (upstream) and control packets (downstream). It will take channel conditions, E-2-E packet TTL, traffic class, packet priority and NACK as input and will decide the necessary reliability measures for that particular information that includes packet storage and packet retrieval.

3) Data Prioritization for Heterogeneous Traffic Support: The purpose of this module is to take care of the heterogeneous traffic flows, from various source ID's $i$, that occur simultaneously in any reference time frame. It will rank and scheduled the packet transmission, having sequence number $s$, by taking into account the packet priority $b$, weighting coefficients $W_{b, s}^{i}$ and E-2-E packet TTL information. It not only helps in scheduled transmissions of the packets but also help in combating challenges posed by the application specific QoS requirements.

Condition 1. Empirically the following values are selected for channel bandwidth sharing:

- For multimedia flow $b=1$, and $W_{b, s}^{i}=0.6$

- For critical scalar information $b=2$ and $W_{b, s}^{i}=0.25$ and

- For less critical scalar information, $b=3$ and $W_{b, s}^{i}=$ 0.15

Condition 2. Empirically the maximum effective rate for multimedia and other scalar motes comes to be:

- Multimedia (High Priority):

$$
W_{b, s}^{i} * \lambda_{\text {Threshold }}=0.6 * \lambda_{\text {Threshold }}
$$


- Scalar critical (Medium Priority):

$$
W_{b, s}^{i} * \lambda_{\text {Threshold }}=0.25 * \lambda_{\text {Threshold }}
$$

- Scalar less critical (Low Priority):

$$
W_{b, s}^{i} * \lambda_{\text {Threshold }}=0.15 * \lambda_{\text {Threshold }}
$$

Condition 3. For any source mote the following condition must be satisfied for the updated estimated rate value:

$\lambda_{e}=\frac{\text { rate in pps } * 512 * 8}{10^{6}}(M b p s) \leq \lambda_{\text {Threshold } * W_{b, s}^{i}}$

Condition 4. Likewise if the parent mote and child motes are all intermediate motes then the link will be shared maximally by the child intermediate mote having highest branch priority i.e.

$$
\sum P_{h}=P_{l} \quad \forall h \epsilon H
$$$$
\text { where, }
$$

$P_{l}=$ effective priority of the intermediate mote ' $l$ ',

$\sum P_{h}=\sum W_{b, s}^{i} \forall h \epsilon H, b \epsilon S, s \epsilon E, i \epsilon D$ is the effective sum of all motes (source, intermediate etc) priorities that are attached to the intermediate mote ' $l$ '.

\section{SIMULATION SETUP AND RESULTS}

NS-2 has been used for evaluating the LCART in a multihop ad-hoc scenario as shown in Figure 2, comprising of 24 motes spaced randomly at an average distance of around 100 meters $(\mathrm{m})$ apart from each other and covering a region of $1000 \times 1000 \mathrm{~m}^{2}$. Motes 0-9 are considered as basic source motes while motes $11-23$ are intermediate motes may or may not have sensing feature and mote 10 acts as Sink. The source motes 1,4 and 7 are considered to be multimedia by nature while source motes $3,5,6$ and $0,2,8,9$ are scalar critical and scalar less critical by nature. The network parameters are listed in the Table 1. The simulation also incorporates the effect of errors introduced by channel interference. The performance of the LCART is evaluated against TCP-WW+[8], TCP-WW[8], TCPNewReno[9] and TCPReno[10].

Good throughput comparison for LCART with other transport layer protocols is shown in Figure 3. As we can see from the graph that LCART exhibits highest good throughput of $0.3112 \mathrm{Mbps}$ in comparison to TCP-WW+ whose good throughput is $0.2874 \mathrm{Mbps}$, TCP-WW whose good throughput is $0.2902 \mathrm{Mbps}$, TCPNewReno and TCP Reno whose good throughputs are 0.2668 and $0.2941 \mathrm{Mbps}$ respectively. The main reason for this good behavior for LCART comes from the protocol efficiency gain from the cross-layer designing which not only best utilizes the network resources for finding the optimal source transmission rates but also helps in quick retrieval of lost packet information. The heart of the LCART, congestion control mechanism, entirely based on the information of TPST, TPIAT, channel loading conditions and intermediate mote $m_{i}$ etc and the cross-layering enables the LCART to effectively monitor these values and pass it to its Kalman based predictor-corrector estimator for finding the optimal source transmission rate. This rate value, which is based on the real time monitored statistics, prevents the unwanted events of packet drop and source transmission rate reduction, thus resulting in high system good throughput.
Figures 4 and 5 shows the average packet drop comparison of LCART against various transport layer protocols. From Figure 4 it is evident that among all protocols only LCART guarantee the successful delivery of high and low priority packet information. It is also evident from Figure 5 that LCART exhibits only $1.0137 \%$ (of the total communication) packet drop in comparison to $2.74 \%, 3.913 \%$ for TCP-WW+ and TCP-WW and $2.47 \%, 2.8 \%$ subsequently for TCPNewReno and TCPReno. The main reason that LCART shows lowest packet drop in comparison to others is because of its transport layer dependency on lower MAC and WirelessPhysical layers which actually feedbacks the transport layer about the channel conditions and the severity of the congestion in the mote's neighborhood. Another reason for this low drop is its stochastically tuned reliability component ( $\beta$ factor), which is based on the link conditions and the nature of the traffic flow, defines the time of storage at local intermediate buffer motes.

Figure 6 shows the average E-2-E data packet latency comparison for various transport layer protocols and the significance of this comparison highlights the use of LCART for WSN targeting heterogeneous traffic simultaneously. From the comparison it is obvious that the LCART outperforms all others by exhibiting the least E-2-E data packet latency for every source. LCART exhibits $<80$ msec E-2-E latency behavior for multimedia information and $<130 \mathrm{msec}$ E-2-E latency for other, non-prior, packet information. TCPReno exhibits worst behavior among all for the high priority information, where as TCP-WW+ shows better response in comparison to TCPWW, TCPNewReno and TCPReno. On average TCP-WW+ and TCP-WW exhibits $>450 \mathrm{msec}$ for high priority sources 2,4 and $>100 \mathrm{msec}$ for high priority source 5 where as similar behavior is being exhibited by TCPNewReno and TCPReno respectively. The reason for this efficient behavior exhibited by the LCART is its fine congestion and reliability control whilst the use of cross-layering the common functionalities of transport and lower layers which keeps E-2-E packet latency to a minimum value. As the rate adjustment is based on Kalman based predictor-corrector estimator which takes lower layer information as input, therefore this control feedback phenomenon helps in achieving the optimal source transmission rate values which keeps network uncongested most of the time thus minimizing the E-2-E data packet latency and packet drop rate caused by congestion resulting in energy efficient design. This optimality also helps LCART in fulfilling the E-2-E data packet latency QoS requirement for multimedia application (e.g. for audio it should be $\leq 150 \mathrm{msec}$ ).

Figure 7 shows the per packet energy consumption (in mili Joules, mJ) comparison of LCART with other transport layer protocols. From Figure 7 it is obvious that the per packet energy consumed by LCART source motes is $\simeq 0.45 \mathrm{~mJ}$ for the entire communication which is high (because of high throughput) in comparison to TCP-WW+, TCP-WW, TCPNewReno and TCPReno which consume per packet energy of 0.4475 $\mathrm{mJ}, 0.4410 \mathrm{~mJ}, 0.3126 \mathrm{~mJ}$ and $0.4394 \mathrm{~mJ}$ respectively. Also it is noticeable that the TCPNewReno has lowest throughput, 


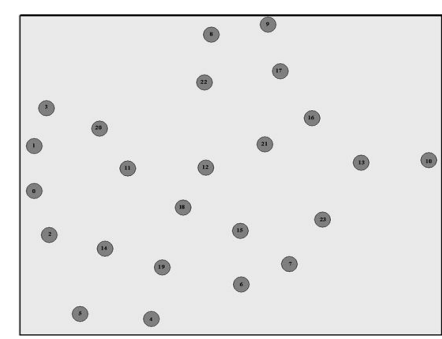

Figure 2. Network topology

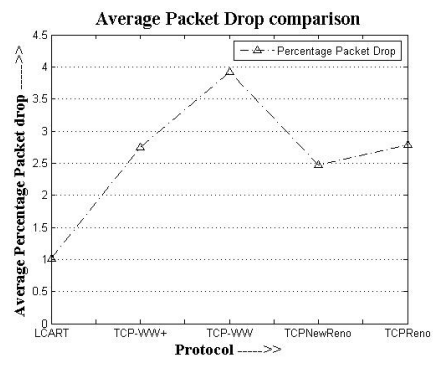

Figure 5. Packet Drop Comparison

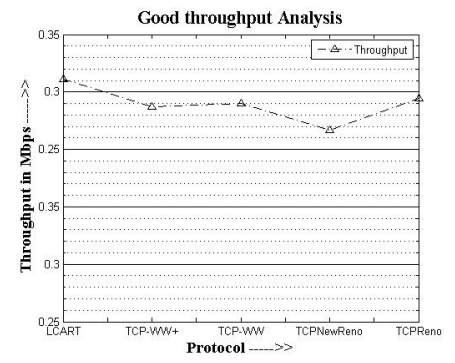

Figure 3. Good Throughput Comparison

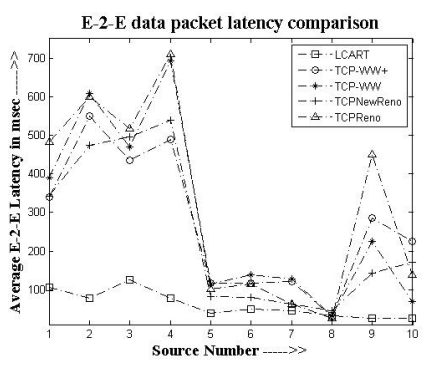

Figure 6.Average E-2-E Packet Latency

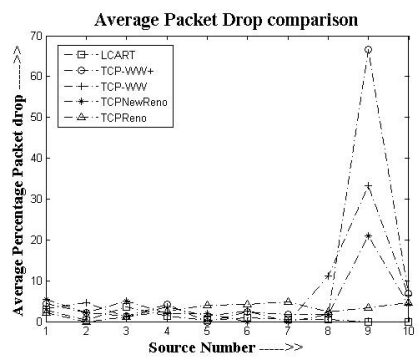

Figure 4. Average Packet Dropped by Sources

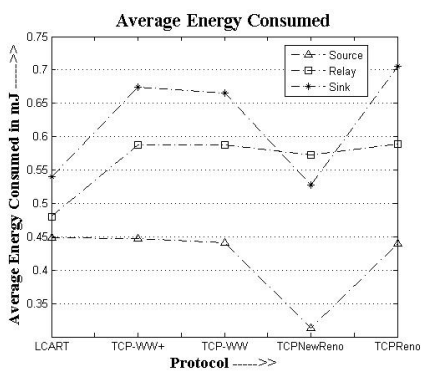

Figure 7. Average Per Packet Energy Consumed source transmission rate is low in comparison to other protocols, and for that the source motes that uses TCPNewReno consumes less per packet energy which is $\simeq 0.3126 \mathrm{~mJ}$.

Also we can see from Figure 7. that the total per packet communication cost for LCART's relay motes is $\simeq 0.4804 \mathrm{~mJ}$ in comparison to $0.5881 \mathrm{~mJ}, 0.5874 \mathrm{~mJ}, 0.5722 \mathrm{~mJ}$ and 0.5894 $\mathrm{mJ}$ for TCP-WW+, TCP-WW, TCPNewReno and TCPReno respectively. Again this confirms the effectiveness of LCART's congestion control and reliability design component that uses cross-layer design feature. Since LCART congestion control mechanism keeps source mote transmission rate to an optimum value, by best utilizing the network resources, therefore it not only prevents the unwanted packet drop due to congestion but also the associated control overhead for retransmissions thus resulting in energy efficiency. Also another good reason for this efficiency is that the LCART reliability module uses stochastically distributed time definition $(\beta)$ for packet storage at intermediate motes, again that time would be governed by the link conditions, nature of traffic flow etc. Similarly we can see from Figure 7 that the effective per packet energy consumed by LCART's sink is minimum $(0.5398 \mathrm{~mJ})$ in comparison to TCP-WW+, TCP-WW, TCPNewReno and TCPReno which consumes $0.674 \mathrm{~mJ}, 0.666 \mathrm{~mJ}, 0.5279 \mathrm{~mJ}$ and $0.706 \mathrm{~mJ}$ respectively. With similar reasoning as for relay motes it is proved that the LCART is the most energy efficient transport layer protocol in comparison to TCP-WW+, TCPWW, TCPNewReno and TCPReno.

\section{CONCLUSION}

In this paper we have envisaged a cross-layered LCART scheme for heterogeneous WSN. We extensively evaluated LCART against TCP-WW+, TCP-WW, TCPNewReno and TCPReno and results reveal that LCART outperforms others in terms of good throughput, average E-2-E data packet latency, average packet drop etc and also showed energy efficient behavior. As a future research path we will incorporate sender based forward packet drop detection scheme and to implement the resulting design into a physical test-bed.

\section{REFERENCES}

[1] Sharif, A., Potdar, V., and Rathnayaka, A. J. D., "Prioritizing Information for Achieving QoS Control in WSN," In the Proceedings of the $24^{\text {th }}$ International Conference on Advanced Information Networking and Applications (AINA), WA, pp. 835-842, 2010.

[2] Pascale, M., and Saoucene, M., "Cross layering in wireless sensor networks," In the Proceedings of the ACM International Conference on Wireless Communications and Mobile Computing: Connecting the World Wirelessly, Germany, pp. 154-159, 2009.

[3] Sharif, A., Potdar, V., and Rathnayaka, A. J. D, "Dependency of Transport layer protocols on the IEEE 802.11 and IEEE 802.15.4 MAC/PHY layer Protocols for WSN: A Step towards Cross-layer design," International Journal of Business Data Communications and Networking (IJDBCN), vol 6(3), 2010, in press.

[4] Cagri, V., Gungor, V. C, Akan, O. B., and Akyildiz., "A real-time and reliable transport $\mathrm{RT}^{2}$ protocol for wireless sensor and actor networks," In the IEEE/ACM Transactions on Networking (TON), USA, pp. 359370, 2008.

[5] Paek, J., and Govindan, R., "RCRT: rate-controlled reliable transport for wireless sensor networks," In the Proceedings of the $5^{t h}$ International conference on Embedded networked sensor systems, Australia, pp. 305 $-319,2007$.

[6] Giancoli, E., Jabour, F., and Pedroza, A., "CTCP: Reliable Transport Control Protocol for Sensor Networks," In the Proceedings of Fourth International Conference on Intelligent Sensors, Sensor Networks and Information Processing, Australia, pp. 493-498, 2008.

[7] Kim, S., Fonseca, R., Dutta, P., Tavakoli, A., Culler, D., Levis, P., Shenker, S., and Stoica, I., "FLUSH: a reliable bulk transport protocol for multihop wireless networks," In the Proceedings of the 5th International conference on Embedded networked sensor systems, Australia, pp. $351-365,2007$.

[8] http://www.cs.ucla.edu/NRL/hpi/tcpw/

[9] Floyd, S. and Henderson, T., "The NewReno Modification to TCP's Fast Recovery Algorithm,” RFC 2582, 1999.

[10] www.ietf.org/rfc/rfc2581.txt 